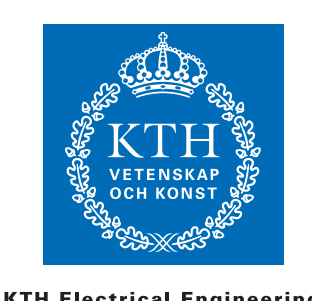

\title{
Uncoded Transmission in Wireless Relay Networks Using Deterministic Modeling
}

(C)2009 IEEE. Personal use of this material is permitted. However, permission to reprint/republish this material for advertising or promotional purposes or for creating new collective works for resale or redistribution to servers or lists, or to reuse any copyrighted component of this work in other works must be obtained from the IEEE.

NICOLAS SCHRAMMAR AND MIKAEL SKOGLUND

Stockholm 2009

Communication Theory Department

School of Electrical Engineering

KTH Royal Institute of Technology

IR-EE-KT 2009:063 


\title{
Uncoded Transmission in Wireless Relay Networks Using Deterministic Modeling
}

\author{
Nicolas Schrammar and Mikael Skoglund \\ School of Electrical Engineering and ACCESS Linnaeus Center \\ KTH, Royal Institute of Technology, Stockholm, Sweden \\ Email: \{nisc, skoglund\}@kth.se
}

\begin{abstract}
We show how communication in the recently proposed deterministic finite field model can be translated into a transmission in the corresponding Gaussian model, using simple uncoded transmission. We find a lower bound on the number of received bits at each node as well as upper and lower bounds on the source-destination rate.
\end{abstract}

\section{INTRODUCTION}

The question of how much information can be sent through a wireless communication network and what the optimal strategy would be to achieve the capacity is crucial for the design of future wireless networks, but appears to be very difficult to answer. Modeling the wireless channel in the most common way as additive white Gaussian noise (AWGN) channel, even the capacity of the three node relay network is still open. Therefore it is reasonable to search for different ways to characterize the transmissions capabilities of wireless networks.

A way to proceed is to change the AWGN channel model for a deterministic channel model [1]. The intuition behind a deterministic model is to emphasize the impact of interference rather than noise on the received signal. It can be shown, that the cut-set upper bound on the capacity is achieved under the deterministic channel model [2]. Furthermore, there is a relation between the capacity under the deterministic model and the AWGN model. The capacity under the AWGN model can be shown to be at most a constant number below the capacity under the deterministic model [3]. Since this constant depends only on the topology of the network, but not on the channel gains, the deterministic model is a good characterization of the AWGN model for large SNR and high capacity.

However, the latter result is found by applying information theoretic arguments and using random coding in the relays. To use the insights of deterministic modeling in realistic scenarios, simple and implementable ways to translate a solution from the deterministic model to the AWGN model have to be found.

In a network that is constrained on uncoded transmission, hierarchical modulation [4] is a way to resolve different information flows. It has been used in a broadcast scenario to convey different information to more than one receivers [5]. The bit error rate of hierarchical QAM constellations has been found in [6].
In this paper we use hierarchical modulation for both transmitting to different nodes in the broadcast case and resolving transmissions from different nodes in the multiaccess case. We achieve this by coordinating the transmission in the deterministic model. Coordinated transmission is related to interference alignment, that has been used on the deterministic model in [7] before. Given that the transmission in the deterministic model is coordinated, we show how to translate it into an easily implementable scheme using uncoded transmission only.

We introduce the channel models and the transmission scheme in Sec. II and Sec. III. The results are presented in Sec. IV, and Sec. V concludes the paper.

\section{Channel Models}

\section{A. AWGN Channel Model}

The complex-valued received signal at node $j=1, \ldots, N$ is modeled as

$$
y_{j}=\sum_{i=1, i \neq j}^{N} h_{i j} x_{i}+z_{j},
$$

where $x_{i} \in \mathbb{C}$ is the signal transmitted at node $i$ and $h_{i j} \in \mathbb{C}$ is the i.i.d. gain of the channel between nodes $i$ and $j$. The noise at node $j$ is i.i.d. complex Gaussian $z_{j} \sim \mathcal{C N}(0,1)$. Each node has power constraint $E\left[x_{i}^{2}\right]=1$.

\section{B. Linear Finite-Field Deterministic Model}

In the deterministic channel model [1] each channel between two nodes $i$ and $j$ has a non-negative integer gain $n_{i j}$. This gain can be interpreted as a number of bits. Define $n_{\max }=$ $\max _{i j} n_{i j}$, so that we can express all received signals $\mathbf{y}_{j} \in$ $\mathbb{F}_{2}^{n_{\max }}$ and transmitted signals $\mathbf{x}_{i} \in \mathbb{F}_{2}^{n_{\max }}$ as vectors of bits. The channel is modeled as

$$
\mathbf{y}_{j}=\sum_{i=1, i \neq j}^{N} \mathbf{S}^{n_{\max }-n_{i j}} \mathbf{x}_{j}
$$

where the summations and multiplications are modulo 2 and

$$
\mathbf{S}=\left[\begin{array}{ccccc}
0 & 0 & 0 & \cdots & 0 \\
1 & 0 & 0 & \cdots & 0 \\
0 & 1 & 0 & \cdots & 0 \\
\vdots & \ddots & \ddots & \ddots & \vdots \\
0 & \cdots & 0 & 1 & 0
\end{array}\right] \in \mathbb{F}_{2}^{n_{\max }} \times \mathbb{F}_{2}^{n_{\max }}
$$


The matrix $\mathbf{S}^{n_{\max }-n_{i j}}$ simply shifts the entries of $\mathbf{x}_{j}$ a number of $n_{\max }-n_{i j}$ positions to the right and fills zeros on the left. Another possible formulation for each of the bits in $\mathbf{y}_{j}$ is

$$
y_{j}[k]=\sum_{i: n_{i j}>n_{\max }-k} x_{i}\left[n_{i j}-n_{\max }+k\right], k=1, \ldots, n_{\max } .
$$

Fig. 1 depicts a deterministic model for a transmission from a source $S$ to a destination $D$ using four relay nodes. For now, consider both solid and dashed lines. The maximum number of bits is chosen $n_{\max }=6$, resembled by six circles for transmission and six circles for reception. The circles are referred to as signal levels. The number of bits $n_{i j}$ is resembled by the number of lines between the nodes, and we refer to these lines as bit-transmissions.

The deterministic model can be related to the corresponding AWGN model by defining [1]

$$
n_{i j}=\left\lceil\log _{2}\left|h_{i j}\right|\right\rceil .
$$

The interpretation of this relations is, that a number $n_{i j}$ of bits can be received above noise level. These bits are assumed to be free of transmission errors. All additional bits, that the transmitter might send, are drowned in noise and convey no information to the receiver.

\section{Generalized Deterministic Model}

The deterministic model can be generalized by defining

$$
n_{i j}=\left\lceil\log _{\vartheta}\left|h_{i j}\right|\right\rceil
$$

for $\vartheta>1$, taking the logarithm to the base $\vartheta$. To keep expressions simple, we will refer to $n_{i j}$ as the number of bits, even though each of those bits carries $\log _{2}(\vartheta)$ bits of information. The set of all bit-transmissions representing a network is denoted by $\mathcal{N}$.

Due to the rounding operation, the translation from deterministic model back to the AWGN model is not explicit,

$$
\vartheta^{n_{i j}-1}<\left|h_{i j}\right| \leq \vartheta^{n_{i j}} .
$$

The ambiguity can be expressed in terms of a random variable $g_{i j} \in \mathbb{C}$

$$
h_{i j}=\vartheta^{n_{i j}} g_{i j},
$$

with $\left|g_{i j}\right| \in\left(\vartheta^{-1}, 1\right]$ and $\arg \left(g_{i j}\right) \in[0,2 \pi)$.

\section{TRANSMISSION SCHEME}

We propose an uncoded transmission scheme, that enables us to translate a transmission in the deterministic channel model to a transmission in the AWGN channel model. Given that the transmission in the deterministic model is coordinated, we can translate it into a transmission in the AWGN model, that uses simple uncoded hierarchical QPSK modulation.

Definition 1. A transmission in the deterministic model is referred to as coordinated if the following constraints are fulfilled.

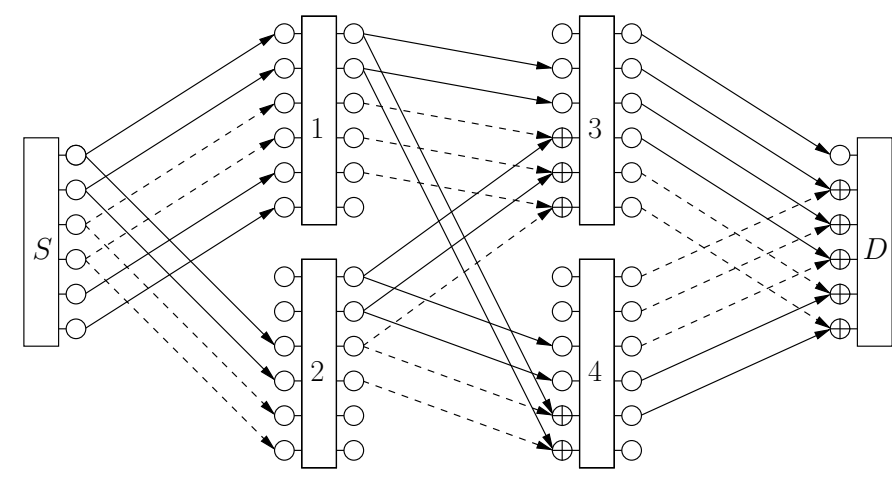

Fig. 1. Example of deterministic model with $n_{\max }=4$ signal levels (circles) and $n_{i j}$ bit-transmissions (lines). Solid lines form a coordinated transmission.

1) A maximum number of one bit is assigned to each signal level at each receiver. Hence, each of the sums in (2) consists of at most one term

$$
y_{j}[k]=x_{i}\left[n_{i j}-n_{\max }+k\right], \quad i \in\left\{i: n_{i j}>n_{\max }-k\right\}
$$

for $j=1, \ldots, N$ and $k=1, \ldots, n_{\max }$.

2) Bit-transmissions from transmitter $i$ to receiver $j$ are assigned in pairs, i.e., a sequence of bits $x_{i}[k], x_{i}[k+1], \ldots, x_{i}[k+l-1]$ has even length $l$.

3) Bit-transmissions form a layered network, that is each path from source to destination has equal length.

A subset of bit-transmissions, that fulfill the constraints of coordination is denoted by $\mathcal{N}_{\text {co }} \subseteq \mathcal{N}$.

Consider Fig. 1 again for an example of a coordinated transmission. The solid lines are used for transmission, whereas the dashed lines are disabled to avoid interference at the receiving nodes. Disabling a bit-transmission is equivalent to sending $x_{i}[k]=0$ on the corresponding signal level. Note that due to the broadcast property, bit-transmissions sent by the same transmitter $i$ at the same signal level $k$ cannot be disabled independently.

The fact that pairs of bits are assigned in a coordinated transmission allows for the description of those pairs in terms of qits. A qit is an entity with four states and is therefore equivalent to a bit pair. According to the deterministic model, a maximum number of

$$
q_{i j}=\left\lfloor\frac{n_{i j}}{2}\right\rfloor
$$

qit-transmissions can be supported by the link from node $i$ to node $j$.

A set $\mathcal{N}_{\text {co }}$ of bit-transmissions that fulfills the constraints of coordination can be expressed in terms of a set $\mathcal{Q}_{\text {co }}$ of qit-transmissions, that resemble the assigned pairs of bittransmissions. Furthermore, we denote the set of all $\mathcal{Q}_{\text {co }}$ as $\mathcal{C O}(\mathcal{N})$

Definition 2. Hierarchical QPSK modulation is a simple modulation strategy to transmit $n_{\text {trans }}$ qits in a single time instance. The transmitted signal of node $i$ is

$$
x_{i}=a \sum_{k=0}^{n_{\text {trans }}-1}\left(\frac{1}{\vartheta}\right)^{2 k} m_{i, k}=\sum_{k=0}^{n_{\text {trans }}-1} x_{i, k},
$$




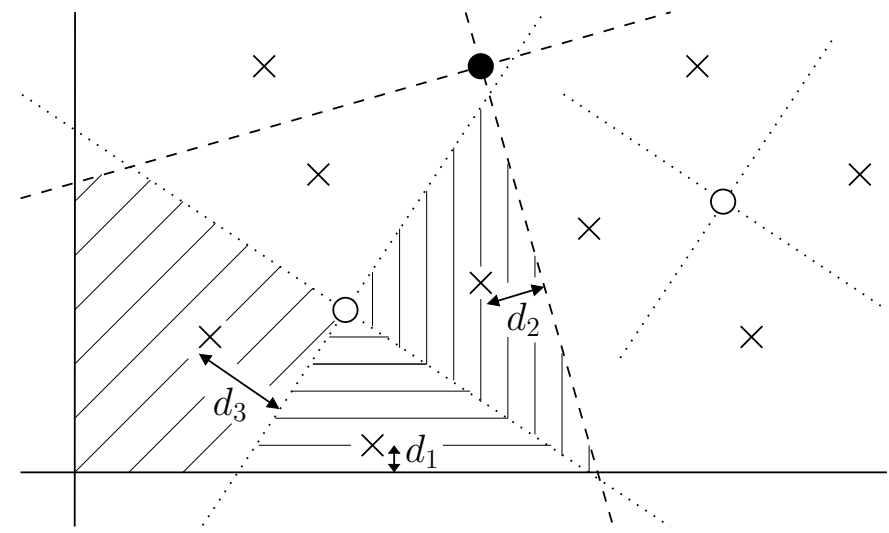

Fig. 2. Part of the signal space of $s_{j}$, consisting of three QPSK constellations. Decision boundaries marked by lines, decisions regions are hatched.

where $m_{k} \in\{1+j, 1-j,-1+j,-1-j\}$ is the $k$-th qit, and $a$ is a constant determined by the power constraint. The interpretation of $x_{i}$ is that of a hierarchical transmission of $n_{\text {trans }}$ nested QPSK constellations $x_{i, k}$.

Due to (1), the received signal $y_{j}$ consists of a superposition of many scaled and rotated QPSK constellations. To facilitate analysis we use the following assumption.

Assumption 1. Assume that the sum

$$
r_{j}=\sum_{i=1, i \neq j}^{N} h_{i j} \sum_{k=q_{i j}}^{n_{\text {trans }}} x_{i, k}
$$

of QPSK constellations $x_{i, k}$, that are received with power below noise power can be regarded as white Gaussian noise with variance $\hat{\sigma}_{j}^{2} \leq N-1$. The resulting model is

$$
y_{j}=\sum_{i=1, i \neq j}^{N} h_{i j} \sum_{k=0}^{q_{i j}-1} x_{i, k}+r_{j}+z_{j}=s_{j}+r_{j}+z_{j} .
$$

In a coordinated transmission the QPSK constellations are received with sufficiently different amplitude to resolve them.

Fig. 2 depicts a part of the signal space of $s_{j}$, that is the noise-free part of the received signal $y_{j}$. The signal consists of three superposed QPSK constellations $c_{i}=h_{i j} x_{i, 1}, i=$ $1,2,3$, with $\mathrm{E}\left[c_{1}^{2}\right]>\mathrm{E}\left[c_{2}^{2}\right]>\mathrm{E}\left[c_{3}^{2}\right]$. The resulting symbols are marked with crosses. To simplify interpretation, the constellation $c_{1}$ is depicted by a black circle, and the sum of the two constellations $c_{1}+c_{2}$ is depicted by white circles.

The receiver resolves the constellations $c_{i}$ successively. The first decisions is about $c_{1}$ and the boundaries are depicted by solid lines. Secondly, the dashed lines are used to decide about $c_{2}$. Finally, $c_{3}$ is resolved by using the dotted lines. The procedure results in heterogeneously shaped decisions regions, highlighted by hatching. In the next step we find a lower bound on the distance between a symbol and its decision boundary.

\section{RESUlts}

\section{A. Number of Received Qits}

Result 1. Consider the noise free part $s_{j}$ of the received signal $y_{j}$ at node $j$, in a system using hierarchical QPSK modulation and coordinated transmission. The minimum distance $d$ between a resolved symbols and the boundary of its decisions region can be lower bounded by

$$
d \geq \frac{\vartheta^{2 q_{\max }}}{\vartheta^{2 q_{\mathrm{res}}}} c
$$

with

$$
c=\sqrt{1 / 2} \sqrt{\frac{\vartheta^{2}+1}{\vartheta^{2}-1}}\left(\vartheta-\sqrt{2}-\frac{1}{\vartheta}\right) .
$$

The distance is a function of the maximum number of qits $q_{\max }=\max _{i} q_{i j}$ that node $j$ can receive and the number of qits $q_{\text {res }} \leq q_{\max }$ that this node chooses to resolve. The proof is found in Appendix A.

The transmission scheme requires the choice of $\vartheta>\frac{1+\sqrt{3}}{\sqrt{2}}$. Observe that $\vartheta$ is chosen too small in the example of Fig. 2. Rotating the white circles counterclockwise reduces $d_{1}$ below zero, but error free detection is only possible if the distance is positive.

Result 2. If we use hierarchical QPSK modulation and coordinated transmission, then under the assumption 1 the number $q_{\text {res }}$ of qits that can be resolved from the received signal $y_{j}$ in (4) below a given target symbol error rate $P_{e}$ can be lower bounded by

$$
q_{\text {res }} \leq q_{\max }-\left\lceil\frac{1}{2} \log _{\vartheta}\left(c^{-1} \sqrt{N \ln \left(P_{e}^{-1}\right)}\right)\right\rceil=q_{\max }-p .
$$

The proof is found in Appendix B.

\section{B. Source Destination Rate}

Result 2 states, that the $p$ least significant qits cannot be resolved below the error rate $P_{e}$. This property can be expressed in terms of a feasible sub network $\mathcal{Q}_{\text {co }}^{p} \subseteq \mathcal{Q}_{\text {co }}$, that does not use any of the $p$ least significant qit-transmissions.

Now, it is simple to formulate an expression for the rate from source $S$ to destination $D$

$$
R=\max _{\mathcal{Q}_{\mathrm{co}}^{p}: \mathcal{Q}_{\mathrm{co}} \in \mathcal{C} \mathcal{O}(\mathcal{N})} \min _{\Omega \in \Lambda_{D}} q\left(\Omega, \mathcal{Q}_{\mathrm{co}}^{p}\right),
$$

measured in qits per transmission. The maximization is over all coordinated transmissions $\mathcal{Q}_{\text {co }} \in \mathcal{C O}(\mathcal{N})$, but using only the feasible part $\mathcal{Q}_{\text {co }}^{p}$ of the network. The argument of the maximization is the maximum flow in the network, that is the number of qit-transmissions $q\left(\Omega, \mathcal{Q}_{\mathrm{co}}^{p}\right)$ in $\mathcal{Q}_{\mathrm{co}}^{p}$ traversing a cut $\Omega$, minimized over all possible cuts $\Lambda_{D}$ separating the destination. Corollary 2.3 in [2] states that the min-cut maxflow is achievable in the deterministic network.

Unfortunately, this maximization is difficult to evaluate, since it requires exhaustive search over all coordinated transmissions. An upper bound on $R$ can be found by coordinating the transmission locally only for a specific cut and minimizing over all cuts. The upper bound can be formulated as

$$
R \leq \min _{\Omega \in \Lambda_{D}} \max _{\mathcal{Q}_{\mathrm{co}}^{p}: \mathcal{Q}_{\mathrm{co}} \in \mathcal{C} \mathcal{O}(\mathcal{N})} q\left(\Omega, \mathcal{Q}_{\mathrm{co}}^{p}\right) .
$$

Furthermore, we propose an algorithm to solve the maximization (5) in a successive way. The solution of the algorithm 


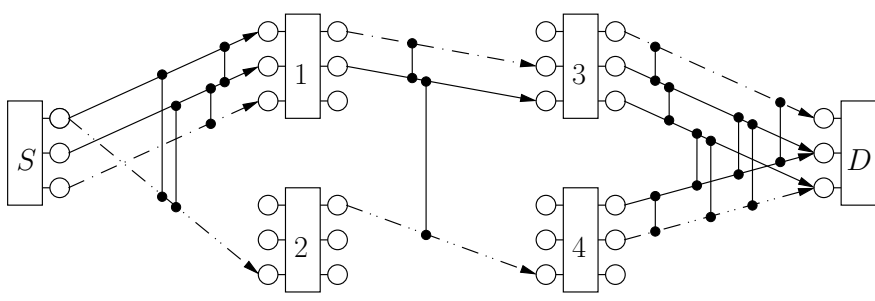

Fig. 3. Representation of the example of Fig. 1 in terms of qit-transmissions Neighbors marked with vertical lines, solutions of algorithm paths marked with dots.

is a lower bound on the source destination rate $R$. For easy formulation, we require the following definition.

Definition 3. Two qit-transmissions are referred to as neighbors, if at most one of them can be used in a coordinated transmission. That is using a specific qit-transmission forbids the use of all its neighbors under the constraints of coordination.

\section{Algorithm paths $(\mathcal{N}, p)$}

1) Initialize all qit-transmissions to feasible, except for the $p$ least significant qit-transmissions of each receiver.

2) For all feasible qit-transmissions, count the number of feasible neighbors.

3) For all links, find the qit-transmission $q(i, j)$ with minimum number $n(i, j)$ of feasible neighbors and set the link cost to this number.

4) Find the least cost path from source to destination, break if no path exists.

5) For all links, set the used qit-transmission $q(i, j)$ and all its neighbors to infeasible.

6) Go to 2).

In each step, the algorithms selects the path of qittransmissions, that forbids the least number of neighboring qittransmissions. The algorithm is designed for layered networks. If the network $\mathcal{N}$ is not layered, the algorithm can be used on a layered subset of $\mathcal{N}$.

Fig. 3 shows the qit-transmissions corresponding to the example network $\mathcal{N}$ depicted in Fig. 1. Each possible pair of bit-transmissions is resembled by a qit-transmissions, that is there exist at most five possible qit-transmissions in each link. However, we assume $p=1$, hence the two least significant bit-transmissions cannot be resolved. Therefore at most three different qit-transmissions can be used in each link. Qittransmissions that are neighbors are connected by a vertical line.

To evaluate the upper bound, it is sufficient to consider the three cuts $\{D\},\{D, 3,4\}$ and $\{D, 1,2,3,4\}$ separating the layers of the network. It is easy to check, that each of the cuts can accomplish at most two qit-transmissions, that are not neighbors of each other. Hence, the upper bound is $R \leq 2$ qits.

The algorithm paths identifies the path marked with one dot as the least cost path in the first loop. After setting the corresponding qit-transmissions and their neighbors to infeasible, there is only one path remaining. It is marked with two dots. Hence, the lower bound is $R \geq 2$ qits, and we have found the source to destination rate $R=2$ qits for this network.

\section{CONCLUSION}

We analyzed an uncoded transmission scheme using hierarchical QPSK modulation to translate solutions from the deterministic to the AWGN channel model. Given a deterministic model of a network, the proposed scheme translates each coordinated transmission in this model into a transmission in the AWGN model. A constant number of $p$ qit-transmissions cannot be resolved under a given target symbol error rate, but this number is independent of the channel coefficients. We found an upper bound on $p$ as well as bounds on the rate $R$ from source to destination.

\section{REFERENCES}

[1] S. Avestimehr, S. N. Diggavi, and D. N. C. Tse, "A deterministic approach to wireless relay networks," in Proc. Allerton Conf. on Communication, Control and Computing, Sep. 2007.

[2] — "Wireless network information flow," in Proc. Allerton Conf. on Communication, Control and Computing, Sep. 2007.

[3] — "Approximate capacity of Gaussian relay networks," in IEEE International Symposium of Information Theory, Jul. 2008.

[4] G. D. Forney, Jr. and G. Ungerboeck, "Modulation and coding for linear Gaussian channels," IEEE Trans. Information Theory, vol. 44, no. 6, pp. 2384-2415, 1998.

[5] T. Wang, A. Cano, G. B. Giannakis, and J. Ramos, "Multi-tier cooperative broadcasting with hierarchical modulations," IEEE Trans. Wireless Communications, vol. 6, no. 8, pp. 3047-3057, 2007.

[6] P. K. Vitthaladevuni and M.-S. Alouini, "Recursive algorithm for the exact BER computation of generalized hierarchical QAM constellations," IEEE Trans. Information Theory, vol. 49, no. 1, pp. 297-307, 2003.

[7] V. R. Cadambe and S. A. Jafar, "Degrees of freedom of wireless networks with relays, feedback, cooperation, and full duplex operation," IEEE Trans. Information Theory, vol. 55, no. 5, pp. 2334-2344, 2009.

\section{APPENDIX}

\section{A. Proof of Result 1}

Under assumption 1, the noise free part of the signal received at node $j$ is

$$
\begin{aligned}
s_{j} & =\sum_{i=1, i \neq j}^{N} h_{i j} \sum_{k=0}^{q_{i j}-1} x_{i, k} \\
& =\sum_{i=1, i \neq j}^{N} \vartheta^{2 q_{i j}} g_{i j} \cdot a \sum_{k=0}^{q_{i j}-1}\left(\frac{1}{\vartheta}\right)^{2 k} m_{i, k} \\
& =a \sum_{i=1, i \neq j}^{N} \sum_{k=0}^{q_{i j}-1} \vartheta^{2 q_{i j}-2 k} g_{i j} m_{i, k} .
\end{aligned}
$$

The application of coordination constrains the entries of the sum. In the deterministic model, coordination assigns pairs of bits to neighboring signal levels at each receiver. Furthermore, no more than one bit is assigned to each signal level. It is easy to verify, that in (6) the exponents $2 q_{i j}-2 k$ of $\vartheta$ equal the signal levels, at which the corresponding qits $m_{k, i}$ are received. Hence, coordination assures, that there is at least a difference of 2 between those exponents. This is due to the assignment of pairs of bits.

Since we are interested in the minimum distance between constellation points, we consider the case, where node $j$ 
receives the maximum number of qits $q_{\max }=\max _{i} q_{i j}$. In this case the exponents of $\vartheta$ are multiples of 2 and we can relabel the sum

$$
s_{j}=a \sum_{i=1}^{q_{\max }} \vartheta^{2 i} g_{i} m_{i}
$$

For ease of notation we drop the index $j$ of the receiving node.

The received signal $s$ consists of $q_{\max }$ scaled and rotated QPSK constellations. We assign a decision region to each of the symbols in the resulting hierarchical constellation (see Fig. 2).

In the first step, we find a lower bound on the minimum distance $d$ between a symbol and the boundary of its decision region, given that the receiver resolves $q_{\text {res }}$ qits. This allows for the formulation of an upper bound on the probability of symbol error

$$
P_{e}=\operatorname{Pr}\left\{\text { one of the } q_{\text {res }} \text { qits is wrong }\right\},
$$

in the proof of Result 2 .

To find the minimum distance, it is convenient to regard each of the receiver's $q_{\text {res }}$ sequential decisions individually. The first decision is about the most significant QPSK constellation in (7), $a \vartheta^{2 q_{\max }} g_{q_{\max }} m_{q_{\max }}$. Without loss of generality we assume $\arg \left(g_{q_{\max }}\right)=0$ and $m_{q_{\max }}=1+j$. Under this assumption the region for correct decision about the most significant QPSK constellation is the first quadrant, though a simple rotation of $s$ would have the same result.

The real and imaginary part of $s$ both carry one bit. To determine the distance to the decision boundary it suffices to consider the real part only. Equivalent expressions can be obtained for the imaginary part. The distance to the decision boundary, i.e. the y-axis, is

$$
\begin{aligned}
d_{1} & =a\left(\left|g_{q_{\max }}\right| \vartheta^{2 q_{\max }}-\sum_{i=1}^{q_{\max }-1} \Re\left\{g_{i} m_{i}\right\} \vartheta^{2 i}\right) \\
& \geq a\left(\frac{1}{\vartheta} \vartheta^{2 q_{\max }}-\sum_{i=1}^{q_{\max }-1} \sqrt{2} \vartheta^{2 i}\right) \\
& =a\left(\frac{1}{\vartheta} \vartheta^{2 q_{\max }}-\sqrt{2} \frac{\vartheta^{2 q_{\max }}-\vartheta^{2}}{\vartheta^{2}-1}\right) \\
& \geq a\left(\frac{1}{\vartheta} \vartheta^{2 q_{\max }}-\sqrt{2} \frac{\vartheta^{2 q_{\max }}}{\vartheta^{2}-1}\right) \\
& =a \vartheta^{2 q_{\max }}\left(\frac{1}{\vartheta}-\frac{\sqrt{2}}{\vartheta^{2}-1}\right) .
\end{aligned}
$$

The first inequality is due to the realization of $g_{i}$ and $m_{i}$ that minimize the distance.

Before the subsequent decision, the receiver will subtract the previously decided part and proceed with the remainder $s-a \vartheta^{2 q_{\max }} g_{q_{\max }} \hat{m}_{q_{\max }}$. We derive the distance to the decision boundary in the $k$-th decision. Again, assume without loss of generality $\arg \left(g_{q_{\max }-k+1}\right)=0$ and $m_{q_{\max }-k+1}=1+j$ to rotate the decision region into the first quadrant. The distance can be bounded

$$
\begin{aligned}
d_{k} & =a\left(\left|g_{q_{\max }-k+1}\right| \vartheta^{2 q_{\max }-k+1}-\sum_{i=1}^{q_{\max }-k} \Re\left\{g_{i} m_{i}\right\} \vartheta^{2 i}\right) \\
& \geq a \vartheta^{2\left(q_{\max }-k+1\right)}\left(\frac{1}{\vartheta}-\frac{\sqrt{2}}{\vartheta^{2}-1}\right)=\underline{d_{k}},
\end{aligned}
$$

using the same arguments.

The minimum possible distance of a symbol to its decision boundary is obtained by minimizing over the distances $d_{k}$

$$
\begin{aligned}
d & =\min _{1 \leq k \leq q_{\text {res }}} d_{k} \geq \min _{1 \leq k \leq q_{\text {res }}} \frac{d_{k}}{\vartheta} \\
& =a \vartheta^{2\left(q_{\max }-q_{\text {res }}+1\right)}\left(\frac{1}{\vartheta}-\frac{\sqrt{2}}{\vartheta^{2}-1}\right) .
\end{aligned}
$$

It remains to find a lower bound on $a$. Recall (3), the transmitted power is

$$
\begin{aligned}
\mathrm{E}\left[x_{i}^{2}\right] & =a^{2} \mathrm{E}\left[\sum_{k=0}^{q_{\text {sent }}-1}\left(\frac{1}{\vartheta}\right)^{2 k} m_{k} \cdot \sum_{l=0}^{q_{\text {sent }}-1}\left(\frac{1}{\vartheta}\right)^{2 l} m_{l}\right] \\
& =a^{2} \sum_{k=0}^{q_{\text {sent }}-1}\left(\frac{1}{\vartheta}\right)^{4 k} \mathrm{E}\left[m_{k}^{2}\right] \\
& =2 a^{2} \frac{1-\vartheta^{-4 q_{\text {sent }}}}{1-\vartheta^{-4}} .
\end{aligned}
$$

The second equation is due to statistical independence of $m_{k}$, that is $\mathrm{E}\left[m_{k} m_{l}\right]=0$ for $k \neq l$. The constraint $\mathrm{E}\left[x_{i}^{2}\right] \stackrel{!}{=} 1$ yields

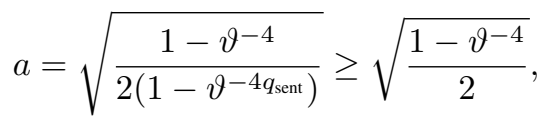

which gives the result.

\section{B. Proof of Result 2}

Due to Assumption 1, the signal $s_{j}$ is corrupted by two AWGN terms $r_{j}$ and $z_{j}$ with variance $\mathrm{E}\left[\left|r_{j}\right|^{2}\right]=\hat{\sigma}_{j}^{2}$ and $\mathrm{E}\left[\left|z_{j}\right|^{2}\right]=1$ respectively. Since $\hat{\sigma}_{j}^{2} \leq N-1$, the sum variance can be lower bounded

$$
\sigma_{\text {sum }}^{2}=\mathrm{E}\left[\left(s_{j}+r_{j}\right)^{2}\right]=\hat{\sigma}_{j}^{2}+1 \leq N .
$$

Given the minimum distance $d$ in the hierarchical constellation, it is straight forward to formulate an upper bound on the probability of symbol error

$$
\begin{aligned}
P_{e} & =\int_{y \notin \Omega} p(y) d y \\
& \leq \int_{|y-s|=d}^{\infty} \frac{1}{\pi \sigma_{\text {sum }}^{2}} \exp \left(-\frac{|y-s|^{2}}{\sigma_{\text {sum }}^{2}}\right) 2 \pi|y-s| d|y-s| \\
& =\exp \left(-\frac{d^{2}}{\sigma_{\text {sum }}^{2}}\right) \leq \exp \left(-\frac{d^{2}}{N}\right) .
\end{aligned}
$$

The upper bound corresponds to the probability, that the received signal $y$ falls outside a circle with radius $d$ around the constellation point $s$. Using result 1 , we arrive at the desired result by simple manipulation. 